\title{
A Comparison of Two Location Models in Optimizing the Decision-Making on the Relocation Problem of Post Offices at Narvik, Norway
}

\author{
Hao Yu, Wei Deng Solvang \\ Department of Industrial Engineering \\ UiT-The Arctic University of Norway \\ Narvik, Norway \\ Email: hao.yu@uit.no; wei.d.solvang@uit.no
}

\begin{abstract}
Since 2013, the postal service provider in Norway: Posten has implemented a new strategic plan to close their large post offices across the country for improving the cost and operational efficiency. Instead, with the cooperation with large retailers, the local postal service is provided at the Posten sub-branches at some supermarkets. In this paper, an optimization problem for relocating the post offices at local supermarkets at Narvik, Norway, is investigated. For resolving the optimization problem, two classical location models: $p$-median problem and maximal covering problem, are employed. The problems are coded and resolved with Lingo optimization solver. The result has provided the optimal relocation plans for the sub-branches of Posten at Narvik with respect to different scenarios. In addition, a comparison between the optimal strategy and the current relocation plan is given in order to show the improvement on the system performance.
\end{abstract}

Keywords - Location problem, maximal covering model, p-median model, decision-making, optimization, operational research

\section{INTRODUCTION}

Since 2013, the postal service provider in Norway: Posten has initiated a new strategic plan for relocating their facilities to local retailing stores and supermarkets for providing the postal service instead of the installation of large post offices. This decision can bring customers for both postal service and shopping at the same place, while, at the same time, it significantly improves the cost saving and operational efficiency for Posten. In this paper, with the help of two location models, we investigated the optimal relocation plans of the post offices at Narvik, Norway, under different scenarios.

When a location decision is made for a facility that provides some kinds of service, e.g. healthcare centers, fuel stations, post offices, etc., it is quite reasonable to consider its convenience so that it should be located within a short proximity to the clients. However, taking into account of operational efficiency, the number of facilities opened is usually with a limitation. Thus, the location problem of such facilities is very complicated, and the objective is to optimize the operational effectiveness and convenience to customers with a finite amount of facilities.

The mathematical models for resolving the decisionmaking problems of facility location have been investigated for more than five decades [1]. The most extensively formulated and focused problems in literature are $p$-median problems and covering problems [2]. This paper employs and compares two well-developed location models in optimizing the relocation problem of the postal system at Narvik.

Section II presents a literature review on the recent applications of $p$-median problem and maximal covering problems. Section III presents a brief introduction of the mathematical models of the two location problems. Section IV presents a case study for the relocation problem of post offices at Narvik. Section V gives a summary of the paper.

\section{LITERATURE REVIEW}

The $p$-median problem and covering location problem have been extensively focused in literature for several decades. Comprehensive literature reviews are given by Farahani, et al. [3], Li, et al. [4], ReVelle and Eiselt [5], Owen and Daskin [6] and Reese [7], with focus on model development, application as well as improvement on solution algorithm. Considering the limited amount of new service points to be installed or opened, covering models are further categorized as set covering problem (without a limitation) and maximal covering problem (with a limitation). In this paper, considering the limitation on the new facilities, the focus of the literature review is given to the recent application of the maximal covering problem in comparison with $p$-median problem on the selection of facility locations.

\section{A. The application of maximal covering problem}

The idea of covering problems was initially mentioned in Berge [8] and Hakimi [9]. Toregas, et al. [10] gave the first mathematical formulation for the set covering problems, while Church and ReVelle [11] first formulated the maximal covering model. Taking into account of the limited amount of new service points to be installed or opened, maximal covering problem has been extensively used for decision-making on the location problems in different fields.

Combining with Geographic Information System (GIS), Curtin, et al. [12] used a maximal covering location model and a new backup covering model to improve the decisions on location problem of the police patrol areas in Dallas, United States. Murawski and Church [13] investigated an improved formulation of the maximal covering model for improving the accessibility of the 
health centers in the rural areas of Suhum District in Ghana. In order to resolve the inflexible capacity problem of traditional covering models, Yin and $\mathrm{Mu}$ [14] investigated a modular capacitated maximal covering problem for the optimization of locations for emergency vehicles. Incorporating with the negative weights. Berman, et al. [15] investigated a maximal covering problem.

For promoting the sustainability related to individual mobility and transport system, bike sharing has becoming increasingly popular in many urban municipalities all over the world. With the help of an improved maximal covering location model, Frade and Ribeiro [16] investigated the optimal bike-sharing stations in Coimbra, Portugal. In order to improve the road control and reduce car accidents, based upon a maximal covering model, Dell'Olmo, et al. [17] developed an multi-period mathematical model for properly allocating the safety cameras in Rome, Italy.

Taking into consideration of the price decisions under the competitive environment, Plastria and Vanhaverbeke [18] improved the traditional maximal covering location problem. Downs, et al. [19] employed the maximal covering problem in order to optimize the decision-making on the installation of wildlife crossing structures in Florida, United States, in order to minimize the animal-vehicle collisions. Considering the uncertainty related to the travel time between different points in a network, Davari, et al. [20] investigated an improved maximal covering location problem with fuzzy parameters.

\section{B. The application of p-median problem}

With a limitation of the amount of new service points to be opened, the $p$-median model aims at minimizing the overall distance travelled by customers. and it was first proposed and studied in Hakimi [21] and Hakimi [9]. In this section, some of the latest development and application of $p$-median location model are presented.

Taking into consideration of the integral constraints on the paths, Lin, et al. [22] developed a general capacitated $p$-hub median problem for the optimizing the cost and operations of a Chinese air cargo network. Considering the uncertainty related to the cost and its probability distribution, Tadei, et al. [23] improved a $p$-median model with stochastic parameters for minimizing the total cost expectation. Goldengorin and Krushinsky [24] investigated the computational performance of a Pseudo-Boolean method for resolving a $p$-median problem. Daskin and Maass [25] focused on the improvement on the computational effectiveness and efficiency of a $p$-median model with heuristics, metaheuristics and Lagrangian heuristics.

For improving the location selection of alternativefuel stations in Orlando, United States, Upchurch and Kuby [26] investigated and compared the performance between a $p$-median model and a flow-refueling model. Karatas, et al. [2] compared the performance of maximal covering problem and $p$-median problem in the network optimization problem with different scenarios of the multiple coverage requirements. Yu, et al. [27] compared the performance of the weighted $p$-median problem and weighted maximal covering problem in optimizing the locations of printers at a university campus so as to improve the efficiency and accessibility.

In this paper, a comparison between classical $p$-median model and maximal covering location problem is presented for optimizing the decision-making of the relocation problem of post offices at Narvik, Norway.

\section{MODEL DESCRIPTIONS}

\section{A. Notations}

The notations blow are used in the mathematical models for both location problems.

\section{Parameters:}

$I, i \quad$ Set and indices of demand points

$J, j \quad$ Set and indices of candidate locations

$h_{i} \quad$ Demand at $i$

$c_{i j} \quad$ Binary parameter determining if $i$ is within

the coverage of $j$

$d_{i j} \quad$ Proximity between $i$ and $j$

$p \quad$ Amount of installed facilities

Decision variables:

$X_{i} \quad$ Binary variable determining if $i$ is within the preferred coverage

$Y_{j} \quad$ Binary variable determining if $j$ is selected

$a_{i j} \quad$ Binary variable determining if $i$ is assigned to $j$

\section{B. The formulation of maximal covering model}

With a limitation on the maximal number of service points to be installed, maximal covering problem aims at the maximization of the demand coverage, as shown in Eq (1a).

Subject to:

$$
\operatorname{Max} \sum_{i \in I} h_{i} X_{i}
$$

$$
\begin{gathered}
X_{i}-\sum_{j \in J} c_{i j} Y_{j} \leq 0, \forall i \in I \\
\sum_{j \in J} Y_{j}=p \\
X_{i}, Y_{j} \in\{0,1\}, \forall i \in I, j \in J
\end{gathered}
$$

Eq. (1b) guarantees, if $X_{i}$ equals to 1 , there is at least one service point is installed within the distance preferred by customers. Eq. (1c) specifies the maximum amount of the service points installed. Eq. (1d) is the requirement of decision variables.

\section{The formulation of $p$-median model}

With a limitation on the amount of service points to be opened, the classical $p$-median location model aims at minimizing total distance travelled from all the customer locations to service points, as shown in Eq. (2a). 


$$
\operatorname{Max} \sum_{i \in I} h_{i} d_{i j} a_{i j}
$$

Subject to:

$$
\begin{gathered}
\sum_{j \in J} a_{i j}=1, \forall i \in I \\
a_{i j} \leq Y_{j}, \forall i \in I, j \in J \\
\sum_{\substack{j \in J \\
Y_{j}}}=p \\
a_{i j}, Y_{j} \in\{0,1\}, \forall i \in I, j \in J
\end{gathered}
$$

Eq. (2b) guarantees the demand from one location is only fulfilled by one facility. Eq. (2c) ensures the customer demand cannot be given to a service point if the candidate location is not selected. Eq. (2d) specifies the number of the facilities to be installed. Eq. (2e) is the requirement of decision variables.

\section{CASE STUDY AND RESULT}

In the case study, both location models are used to optimize the decision-making of the post office relocation problem at Narvik, Norway.

\section{A. Generation of parameters}

Narvik is the second largest municipality in Nordland County, northern Norway, and the total population is 18,756 in accordance with the Statistic Norway 2017. In this study, the central urban area of Narvik is divided into 40 sub-areas on Google map, as shown in Fig. 1 (areas A1, $\mathrm{A} 2, \ldots, \mathrm{E} 8)$. For the simplicity sake, the following assumptions in modeling and optimization are first made:

- Each sub-area is considered as one customer area, and all the customer demands from one sub-area are aggregated at its center point.

- The customer demands for postal services are directly proportional to the population of each sub-area.

- Throughout the urban residential area of Narvik, the population follows uniform distribution.

Based upon the aforementioned assumptions, the relative customer demands of each sub-area is directly proportional to the portion of its residential area and can then be estimated. It is noteworthy that some sub-areas with very few residences (e.g. A1, D8, E2, etc.) are eliminated from the analysis, and in total 27 demand points are investigated in this study.

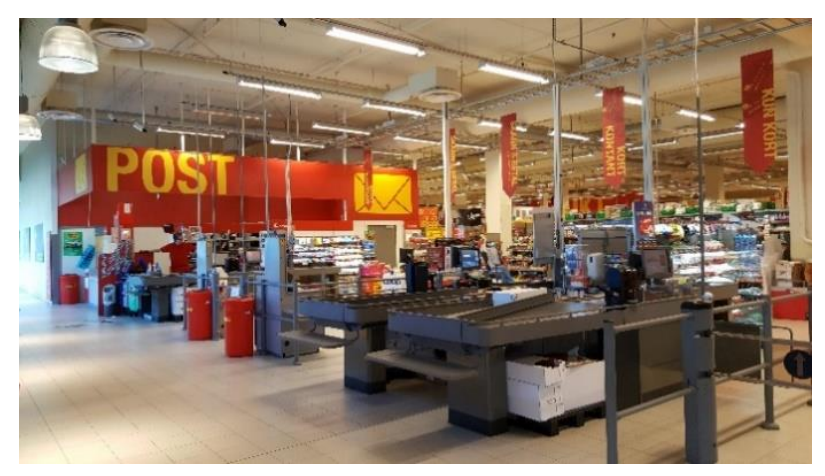

Fig. 2. Post service provided at Coop Extra in Narvik.

Before the relocation of the post offices, there was one large post office at a shopping mall in the city center (area C5). In 2013, the large post office was closed and replaced by the sub-offices at two supermarkets: Spar (area B5) and Coop Extra (D3). Fig. 2 shows the postal services provided at Coop Extra in Narvik. In this paper, the objective is to assess and optimize, through implementing both location

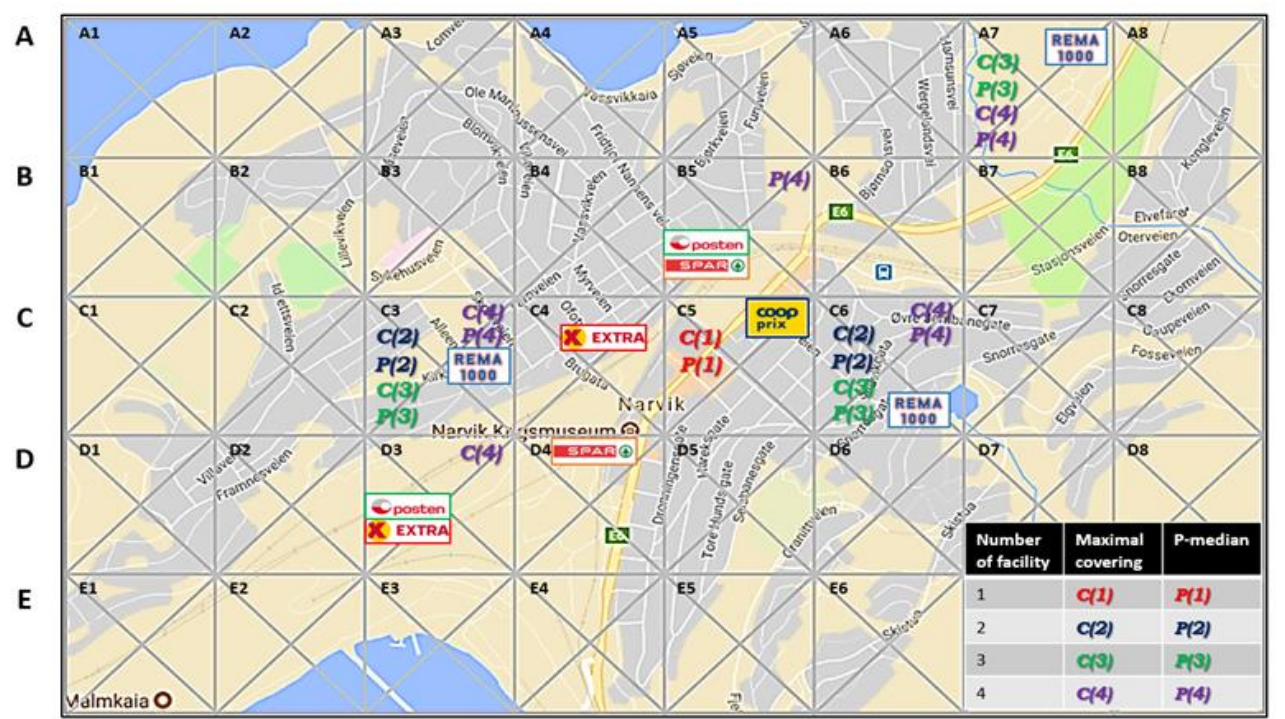

Fig. 1. Customer demand points, candidate locations for post offices and optimal decisions with respect to different scenarios. 
models, the performance of the relocation decisions. It is assumed that all the supermarkets at the urban area of Narvik can be considered as the candidate locations for relocating post offices. As can be seen in Fig. 1, there are eight supermarkets ( 8 candidate locations) including three Rema 1000, two Spar, two Coop Extra and one Coop Prix located at areas A7, B5, C3, C4, C5, C6, D3 and D4, respectively.

Due to the urban context of the studied area, the proximity between customer locations and candidates for post offices is estimated by rectilinear distance metric $\left(d_{(x, y)}=\left|x_{1}-x_{2}\right|+\left|y_{1}-y_{2}\right|\right)$ on the map. In addition, the coverage matrix should be determined when maximal covering problem is investigated. For estimating the customer preferred proximity to post office (coverage), a random survey is given to more than 100 people for investigating their preferred distance for having the postal service, and the result shows the preferred coverage distance of a post office is $0.9 \mathrm{~km}$ in this case. Based on the distance matrix $d_{i j}$ and coverage distance of post offices, the coverage matrix $c_{i j}$ can then be determined.

\section{B. Result and discussion}

The decision-making problem is programmed with Lingo solver. Because of the problems tested are relatively small, all the optimizations can be done within 2 seconds. We found out the optimal solutions for both location models with respect to the changing $p$ from 1 to 8 . Fig. 1 shows the optimal selection of facilities of both location models in the first four scenarios.

As shown, when $p$ increases from 1 to 3 , both $p$ median problem and maximal covering problem generate same optimal solutions. When only one post office is to be opened, the optimal location is as same as the original location of the large post office at the shopping mall located at C5.

TABLE I

PERFORMANCE COMPARISON BETWEEN THE CURRENT PLAN AND THE OPTIMAL PLAN

\begin{tabular}{lll}
\hline$p=2$ & \multicolumn{2}{l}{ Evaluation indicators } \\
\cline { 2 - 3 } & Maximal demand & Minimal overall \\
& coverage & distance travelled \\
\hline Current plan & 12,765 & 15,092 \\
Optimal plan & 14,840 & 12,635 \\
Improvement & $16.26 \%$ & $16.28 \%$ \\
\hline
\end{tabular}

When $p$ increases to 2, the optimal solutions for both location models are C3 and C6, which are different from the current relocation plan at B5 and D3. Table I shows the performance comparison between the current relocation plan and the optimal one. As shown, compared with the current relocation plan, both maximal coverage of customer demands and minimal overall distance travelled by all customers can be improved by approximately $16.3 \%$ in the optimal relocation plan. Further, it is also noteworthy that, compared with the setting up of the contracts and training schemes with two different retailers (Spar and Coop) in the current relocation plan, the optimal solution provides a simplified contracting and training process with only one retailer: Rema 1000. In addition, contracting with Rema 1000 may further benefit Posten in the future expansion when $p$ increases to 3, as illustrated in Fig. 1.

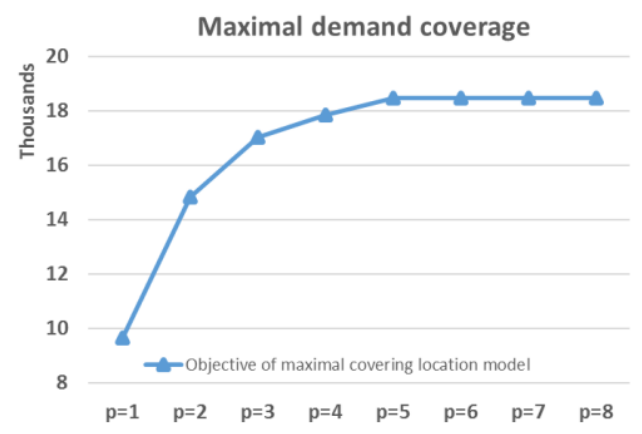

Fig. 3. Maximal demand coverage with respect to the increase on facilities to be opened

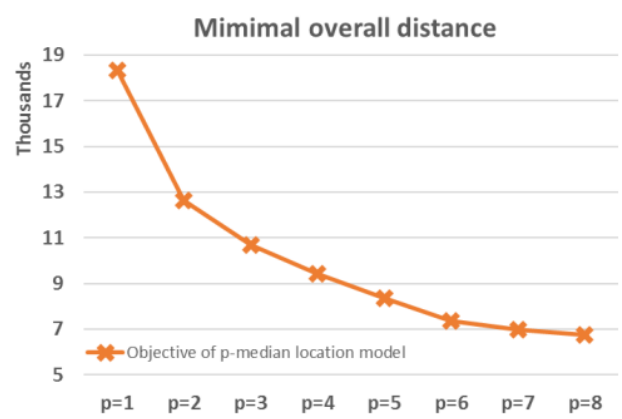

Fig. 4. Minimal overall distance travelled with respect to the increase on facilities to be opened.

When $p$ continues to increase, the optimal solutions of $p$-median model and maximal covering model become different. Figs. 3 and 4 illustrate the change of maximal demand coverage and minimal overall distance with respect to the increase on facilities to be opened from 1 to 8. As shown in Fig. 3, the maximal demand coverage increases gradually until $p$ reaches 5 (bottleneck), from which the increase on the number of facilities will not lead to an improvement on demand coverage. However, from Fig. 4, the overall distance travelled by all customers can be continuously reduced with the increase on the number of post offices opened at Narvik. Furthermore, the improvement on both performance indicators is much more effective at the beginning stage from $p=1$.

\section{CONCLUSION}

In this paper, with the help of two location models, we investigated the optimal relocation plan of post offices at Narvik, Norway. From the analysis of the computational results, both customer demand coverage and overall distance travelled by all customers can be improved with the optimal relocation plan. Moreover, the contracting and 
training process may also be simplified based on the cooperation with only one retailer in the optimal solution. The research work has illustrated, through a real-world case study, the application of mathematical programming and operational research methods for supporting a complex decision-making problem.

\section{ACKNOWLEDGMENT}

This research is supported by Optimization/Reoptimization of Digital, Customer-Focused, Sustainable and Low-Carbon Logistics and Transportation Network in Industry 4.0 (OptiLog 4.0) project. The project is financed under Transport 2025 (No. 283084) programme by The Research Council of Norway.

\section{REFERENCES}

[1] S. García and A. Marín, "Covering location problems," in Location Science: Springer, 2015, pp. 93-114.

[2] M. Karatas, N. Razi, and H. Tozan, "A Comparison of p-median and Maximal Coverage Location Models with Q-coverage Requirement," Procedia Engineering, vol. 149, pp. 169-176, 2016.

[3] R. Z. Farahani, N. Asgari, N. Heidari, M. Hosseininia, and M. Goh, "Covering problems in facility location: A review," Computers \& Industrial Engineering, vol. 62, no. 1, pp. 368-407, 2012.

[4] X. Li, Z. Zhao, X. Zhu, and T. Wyatt, "Covering models and optimization techniques for emergency response facility location and planning: a review," Mathematical Methods of Operations Research, vol. 74, no. 3, pp. 281-310, 2011.

[5] C. S. ReVelle and H. A. Eiselt, "Location analysis: A synthesis and survey," European journal of operational research, vol. 165, no. 1, pp. 1-19, 2005.

[6] S. H. Owen and M. S. Daskin, "Strategic facility location: A review," European journal of operational research, vol. 111, no. 3, pp. 423-447, 1998.

[7] J. Reese, "Solution methods for the $\mathrm{p}$ - median problem: An annotated bibliography," Networks, vol. 48, no. 3, pp. 125-142, 2006.

[8] C. Berge, "Two theorems in graph theory," Proceedings of the National Academy of Sciences, vol. 43, no. 9, pp. 842-844, 1957.

[9] S. L. Hakimi, "Optimum distribution of switching centers in a communication network and some related graph theoretic problems," Operations research, vol. 13, no. 3, pp. 462-475, 1965.

[10] C. Toregas, R. Swain, C. ReVelle, and L. Bergman, "The location of emergency service facilities," Operations research, vol. 19, no. 6, pp. 1363-1373, 1971.

[11] R. Church and C. ReVelle, "The maximal covering location problem," in Papers of the Regional Science Association, 1974, vol. 32, no. 1, pp. 101-118: Springer.

[12] K. M. Curtin, K. Hayslett-McCall, and F. Qiu, "Determining optimal police patrol areas with maximal covering and backup covering location models," Networks and Spatial Economics, vol. 10, no. 1, pp. 125-145, 2010.

[13] L. Murawski and R. L. Church, "Improving accessibility to rural health services: The maximal covering network improvement problem," SocioEconomic Planning Sciences, vol. 43, no. 2, pp. 102110, 2009.

[14] P. Yin and L. Mu, "Modular capacitated maximal covering location problem for the optimal siting of emergency vehicles," Applied Geography, vol. 34, pp. 247-254, 2012.

[15] O. Berman, Z. Drezner, and G. O. Wesolowsky, "The maximal covering problem with some negative weights," Geographical analysis, vol. 41, no. 1, pp. 3042, 2009.

[16] I. Frade and A. Ribeiro, "Bike-sharing stations: A maximal covering location approach," Transportation Research Part A: Policy and Practice, vol. 82, pp. 216227, 2015

[17] P. Dell'Olmo, N. Ricciardi, and A. Sgalambro, "A multiperiod maximal covering location model for the optimal location of intersection safety cameras on an urban traffic network," Procedia-Social and Behavioral Sciences, vol. 108, pp. 106-117, 2014.

[18] F. Plastria and L. Vanhaverbeke, "Maximal covering location problem with price decision for revenue maximization in a competitive environment," $O R$ spectrum, vol. 31, no. 3, pp. 555-571, 2009.

[19] J. Downs, M. Horner, R. Loraamm, J. Anderson, H. Kim, and D. Onorato, "Strategically locating wildlife crossing structures for Florida panthers using maximal covering approaches," Transactions in GIS, vol. 18, no. 1, pp. 46-65, 2014.

[20] S. Davari, M. H. F. Zarandi, and A. Hemmati, "Maximal covering location problem (MCLP) with fuzzy travel times," Expert Systems with Applications, vol. 38, no. 12, pp. 14535-14541, 2011.

[21] S. L. Hakimi, "Optimum locations of switching centers and the absolute centers and medians of a graph," Operations research, vol. 12, no. 3, pp. 450-459, 1964

[22] C.-C. Lin, J.-Y. Lin, and Y.-C. Chen, "The capacitated p-hub median problem with integral constraints: An application to a Chinese air cargo network," Applied Mathematical Modelling, vol. 36, no. 6, pp. 2777-2787, 2012.

[23] R. Tadei, N. Ricciardi, and G. Perboli, "The stochastic p-median problem with unknown cost probability distribution," Operations Research Letters, vol. 37, no. 2, pp. 135-141, 2009.

[24] B. Goldengorin and D. Krushinsky, "A computational study of the pseudo-Boolean approach to the p-median problem applied to cell formation," in Network Optimization: Springer, 2011, pp. 503-516.

[25] M. S. Daskin and K. L. Maass, "The p-median problem," in Location science: Springer, 2015, pp. 2145.

[26] C. Upchurch and M. Kuby, "Comparing the p-median and flow-refueling models for locating alternative-fuel stations," Journal of Transport Geography, vol. 18, no. 6, pp. 750-758, 2010.

[27] H. Yu, W. D. Solvang, and J. Yang, "Improving Accessibility and Efficiency of Service Facility through Location-based Approach: A Case Study at Narvik University College," Advanced Materials Research, no. 1039, 2014. 